\title{
Design of the Robust Controller of the APT Fine Tracking System Based on the Structured Singular Value Theory
}

\author{
Mingqiu Li \& Hongzuo Li \\ Institute of Electronic and Information Engineering, Changchun University of Science and Technology \\ Changchun 130022, China
}

Tel: 86-431-8558-2437_E-mail:limingqiu2003@126.com

\begin{abstract}
APT (Acquisition, Pointing, Tracing) system is the important component of the space laser communication system, and its tracing accuracy is mainly determined by the fine tracing subsystem. To enhance the tracing accuracy and robustness of the APT system, the robust control technology based on the structured singular value theory ( $\mu$ integration) is adopted in the design of the controller of the APT fine tracing subsystem, and the mathematical model of the augmented object of the fine tracing subsystem based on $\mu$ integration is established, and the robust $H_{\infty}$ controller is designed by the $\mathrm{D}-\mathrm{K}$ iterative method in this article, and the bandwidth of the fine tracing subsystem could achieve $318 \mathrm{~Hz}$, which could satisfy the requirements of the APT system to the bandwidth of the fine tracing subsystem. The equivalent sine simulation of the system indicates that the system tracing accuracy is better than $0.6 \mu \mathrm{rad}$.

Keywords: Laser communication, Compound axis system, Fine tracing subsystem, Robust control, $\mu$ integration

\section{Components of the APT system}

The components of the compound axis APT system are seen in Figure 1, and the coarse tracing subsystem includes the gimbal servo turntable and the coarse-tracing CCD, with the characteristics such as wide dynamic range, narrow control bandwidth, lower resonance frequency, and it could accomplish the initial orientation of the laser axis and realize the capture and coarse tracing. The main components of the fine tracing subsystem are the fast steering mirror and the fine tracing detector, and the fine tracing subsystem could further correct the residual error which could not be compensated by the coarse tracing loop, to satisfy the aiming and tracing accuracy required by the system (Toyoda Masahiro, 2006, P.4-12 \& Tzung-Hsien Ho, 2005, P.81-92 \& T. Tolker-nielsen, 2002, P.6-20 \& Liu, 2006, P.103-103).
\end{abstract}

\section{Mathematical model of the fine tracing subsystem}

The executive component of the fine tracing subsystem is the two-dimensional fast steering mirror, and its mathematical model is

$$
G_{F}(s)=\frac{\omega_{n}^{2}}{s^{2}+2 \zeta \omega_{n} s+\omega_{n}^{2}}=\frac{8.2 \times 10^{7}}{s^{2}+1.3 \times 10^{4} \times s+8.2 \times 10^{7}}
$$

The fine tracing imaging system is the CCD camera with the frame frequency of $1600 \mathrm{~Hz}$, and its mathematical model could equal a delay factor, and the delay time is determined by the frame frequency of the fine tracing CCD camera.

\section{Design of the robust controller based on the structured singular value theory ( $\mu$ integration) \\ 3.1 Establishment of the fine tracing system augmented object model based on $\mu$ integration}

The robust control structure of the fine tracing subsystem is seen in Figure 2, and $K(s)$ is the transfer function of the system controller, and $\theta_{i}$ is the target visual axis, and $\theta_{o}$ is the visual axis of fine tracing. $d_{1}$ is the main disturbance acting on the visual axis of fine tracing, $d_{2}$ and $z_{2}$ are evaluation signals of the system to the multiplicative perturbing robustness, $z_{3}$ is the evaluation signal to the control signal of system, $z_{1}$ is the evaluation signal to the response of the system to the disturbance, $W_{1}$ is the weighted function to measure the disturbance performance of the system, $W_{2}, W_{3}$ and $W_{4}$ respectively are the weighted functions of multiplicative perturbing, disturbance perturbing, and control signal. $\Delta(s)$ is multiplicative perturbing, and $\|\Delta\|_{\infty}<1$. The augmented object model of the fine tracing system based on $\mu$ integration obtained from Figure 
2 is seen in Figure 3.

\subsection{Selection of the weighted function}

The selection of the weighted function is the core approach of the robust controller design based on $\mu$ integration, and the weighted function determines the robust stability and the robust tracing performance of the system, and through repeated adjustment, the weighted function is

$$
\begin{aligned}
& \omega_{1}(s)=\frac{0.782 s+100}{s+0.00009} \\
& \omega_{2}(s)=\frac{33.3 s^{3}+3.567 \times 10^{5} s^{2}+1.9 \times 10^{9} s+5.417 \times 10^{12}}{s^{3}+3.967 \times 10^{4} s^{2}+4.042 \times 10^{8} s+6.708 \times 10^{12}} \\
& \omega_{3}(s)=0.24 \\
& \omega_{4}(s)=\frac{9.98 s+1.412 \times 10^{4}}{s+1.298 \times 10^{5}} \\
& 3.3 \text { The } \mu \text { integration of controller }
\end{aligned}
$$

The design target of the robust controller based on $\mu$ integration is to design the robust $H_{\infty}$ controller $K(s)$ by the $\mu$ theory, and minimize the $\mu$ value of the closed-loop transfer function $F(G, K)$ from $d$ to $z$, i.e. minimize $\mu_{\Lambda}(M(j \omega))$, and $M(s)=F(G, K)$ (Mei, 2008, P.96-100).

When designing the controller of the APT fine tracing subsystem by the D-K iterative method, the value of $\mu$ obtained through three times of iteration equals the norm of $H_{\infty}$, and the iterative process ends. The order of controller and the $\mu$ values of the corresponding controllers are seen in Table 1.

From Table 1, the values of $\mu$ of the third iteration and the second iteration are very close with the norm of $H_{\infty}$, because the order of the controller obtained in the third iteration is higher. So the result of the second iteration is the final controller, and the relationship between the structured singular value and the frequency is seen in Figure 4, and the peak value of $\mu$ is 0.413 , and the mathematical model of the 13 -order controller after two iterations is

$$
\begin{aligned}
& K(s)=\frac{914.669(\mathrm{~s}+6 \mathrm{e} 6)(\mathrm{s}+1.35 \mathrm{e} 5)(\mathrm{s}+3.36 \mathrm{e} 4)^{\wedge} 2(\mathrm{~s}+724.6)(\mathrm{s}+347.2)}{(\mathrm{s}+6.001 \mathrm{e} 006)(\mathrm{s}+3.458 \mathrm{e} 004)(\mathrm{s}+3.264 \mathrm{e} 004)(\mathrm{s}+6152)(\mathrm{s}+1868)(\mathrm{s}+638.8)} \\
& \cdot \frac{(\mathrm{s}+309.6)(\mathrm{s}+289.5)\left(\mathrm{s}^{\wedge} 2+6087 \mathrm{~s}+1.998 \mathrm{e} 008\right)^{\wedge} 2}{(\mathrm{~s}+309.5)^{\wedge} 2(\mathrm{~s}+0.0001)\left(\mathrm{s}^{\wedge} 2+5760 \mathrm{~s}+1.939 \mathrm{e} 008\right)\left(\mathrm{s}^{\wedge} 2+6475 \mathrm{~s}+2.056 \mathrm{e} 008\right)} \\
& \text { 3.4 Analysis of the simulation of the APT fine tracing system based on robust } H_{\infty} \text { controller }
\end{aligned}
$$

Through designing the robust controller by the $\mu$ theory, the step response and the closed-loop frequency characteristic curve of the fine tracing system respectively are seen in Figure 5 and Figure 6, and the bandwidth of the fine tracing subsystem is $2000 \mathrm{rad} / \mathrm{s}(318.3 \mathrm{~Hz})$, and the adjustment time is $0.0045 \mathrm{~s}$, and the overshoot is zero, which all could satisfy various index requirements of the system.

\section{Simulation of the tracing performance of the compound axis APT system}

The simulation analysis of the tracing performance of the APT system is implemented by adding equivalent sin signal to the APT signal. According to the maxim work speed and the accelerated speed required by the system, the amplitude of the equivalent sin signal is $0.0262 \mathrm{rad}$, and the frequency is $2 \mathrm{rad} / \mathrm{s}(\mathrm{Hu}, 2005, \mathrm{P} .7-9 \& \mathrm{Fu}, 1998$, P.5-10 \& Wu, 2008, P.41-47).

From the simulation result, the responses of the APT system based on the robust control and the system based on the routine compensation respectively are the curve 1 and the curve 2 in Figure 7 . The part error enlargement figure of the APT system based on the robust control is Figure 8. The result shows that the robust control based on $\mu$ integration could enhance the tracing accuracy of the system, and the tracing accuracy of the system is better than $0.6 \mu \mathrm{rad}$.

\section{Conclusions}

In this article, the $\mu$ integration method is used to design the robust $H_{\infty}$ controller of the APT fine tracing subsystem, which could make the system bandwidth achieve $318 \mathrm{~Hz}$. By the equivalent sin simulation of the APT system, the result shows that robust control technology based on $\mu$ integration could make the tracing accuracy of the fine tracing system exceed $0.6 \mu \mathrm{rad}$. 


\section{References}

$\mathrm{Fu}$, Chengyu, Ma, Jiaguang \& Ye, Buxia et al. (1998). Application of the Compound Axis Control System. Opto-Electronic Engineering. No.25(4). P.5-10.

$\mathrm{Hu}$, Qinggong \& Wang, Kejia. (2005). Design 0f atmosphere laser communication APT system and fine tracking system simulation. Application science and technology. No.32(12). P.7-9.

Liu, Ximin, Sun, Liren \& Sun, Jianfeng. (2006). Bandwidth Design of Composite Axis System in Satellite Laser Communication. Acta Optica Sinica. No.26(1). P.103-103.

Mei, Shengwei, Shen, Tielong \& Liu, Kangzhi. (2008). Theory and Application of Modern Robust Control. Beijing: Tsinghua University Press. P.96-100.

Wu, Qiong. (2008). Study on the Fine Tracking Control Technology of the Laser Communication APT System in Free Space. Changchun: Master's Degree Thesis of Changchun University of Science and Technology. P.41-47.

Toyoda Masahiro. (2006). Acquisition and tracking control of satellite-borne laser communication systems and simulation of downlink fluctuations. Optical Engineering (S0091-3286). No.45(3). P.4-12.

T. Tolker-nielsen, G. Oppenhauser. (2002). In-orbit test result of an operational optical inter-satellite link between ARTEMIS and SPOT4,SILEX.Proc.SPIE. Vol.4635. P.6-20.

Tzung-Hsien Ho, Stuart D. Milner, Christopher C Davis. (2005). Fully optical real-time pointing, acquisition and tracking system for free space optical link. Proc.SPIE. Vol.5712. P.81-92.

Table 1. D-K iterative result

\begin{tabular}{|c|c|c|c|}
\hline Iterative times & Orders of controller & $\mu$ & $H_{\infty}$ Norm \\
\hline 1 & 10 & 0.488 & 1.598 \\
\hline 2 & 13 & 0.409 & 0.413 \\
\hline 3 & 16 & 0.396 & 0.396 \\
\hline
\end{tabular}

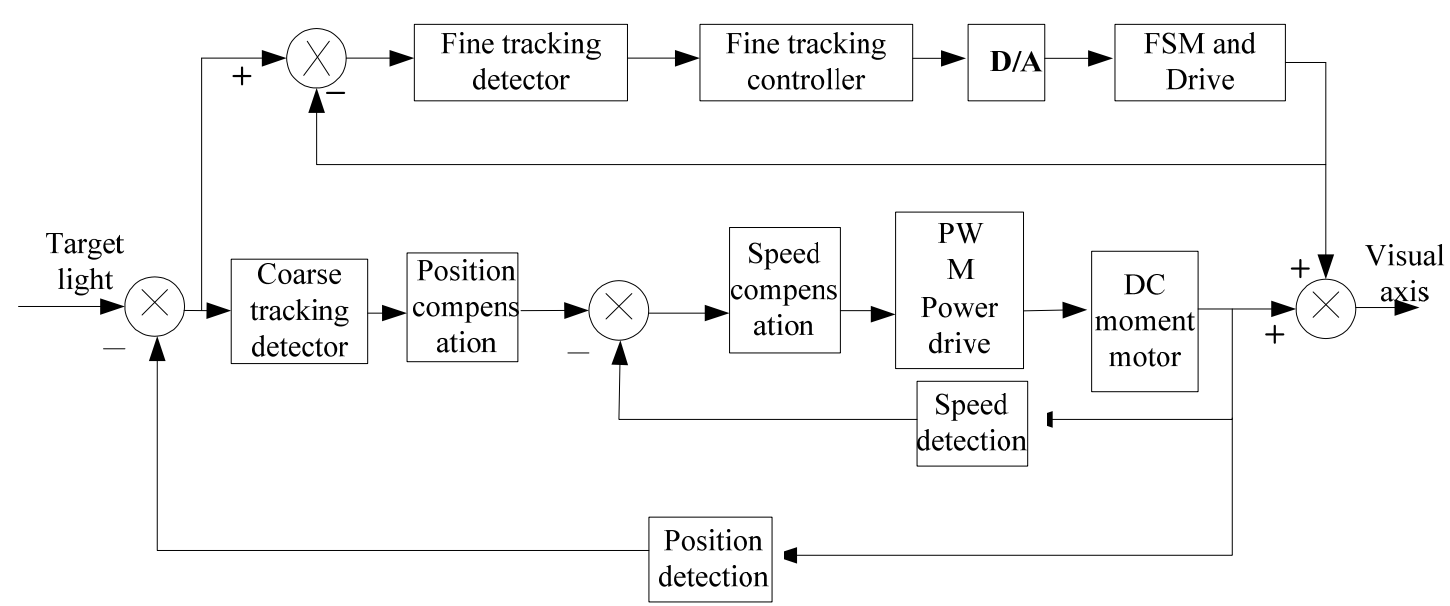

Figure 1. Block Diagram of the APT System

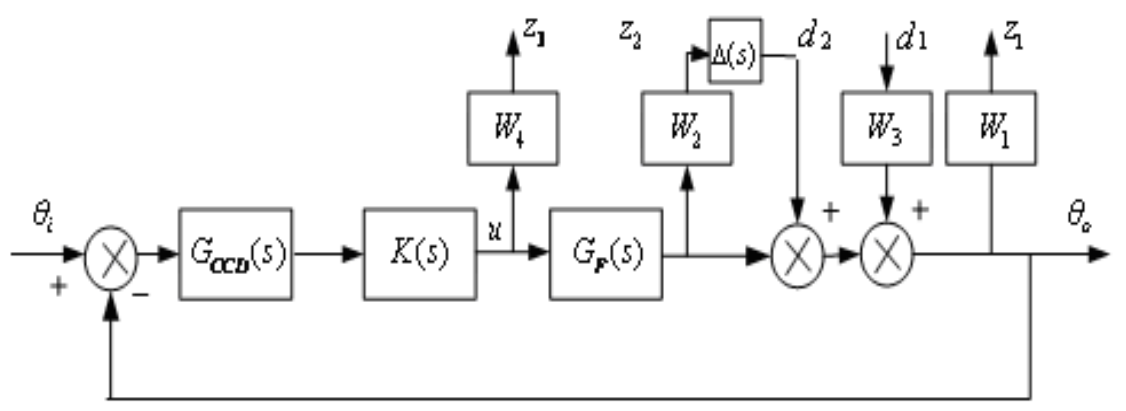

Figure 2. Robust Control Structure of the Fine Tracking Subsystem 


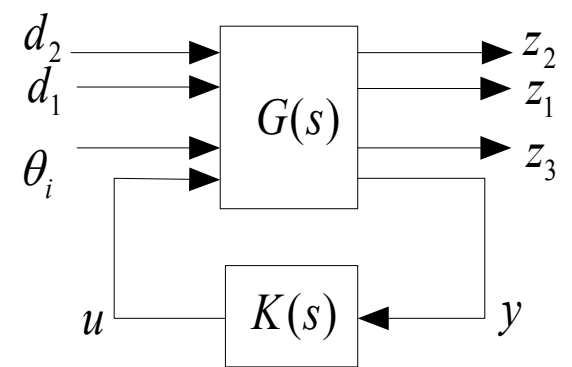

Figure 3. Augmented Object Model of the Fine Tracking System

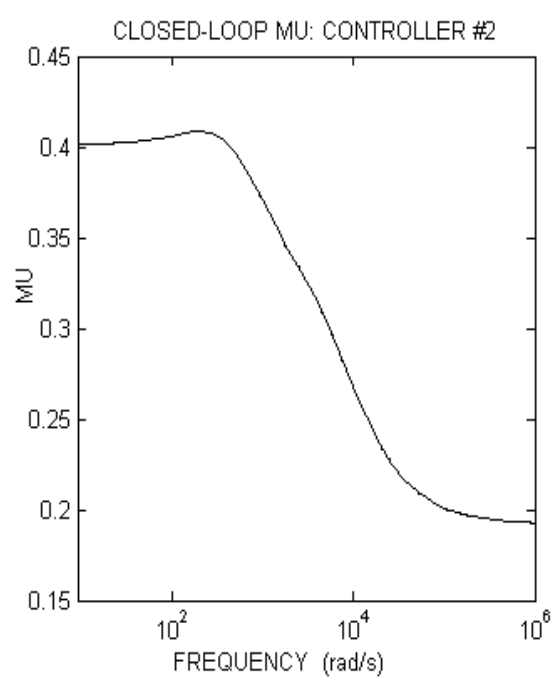

Figure 4. Structured Singular Value of the System

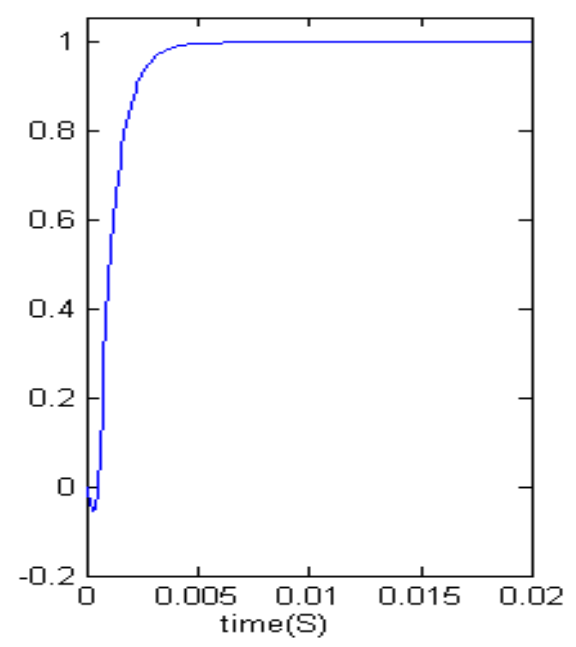

Figure 5. Step Response of the Fine Tracking System 


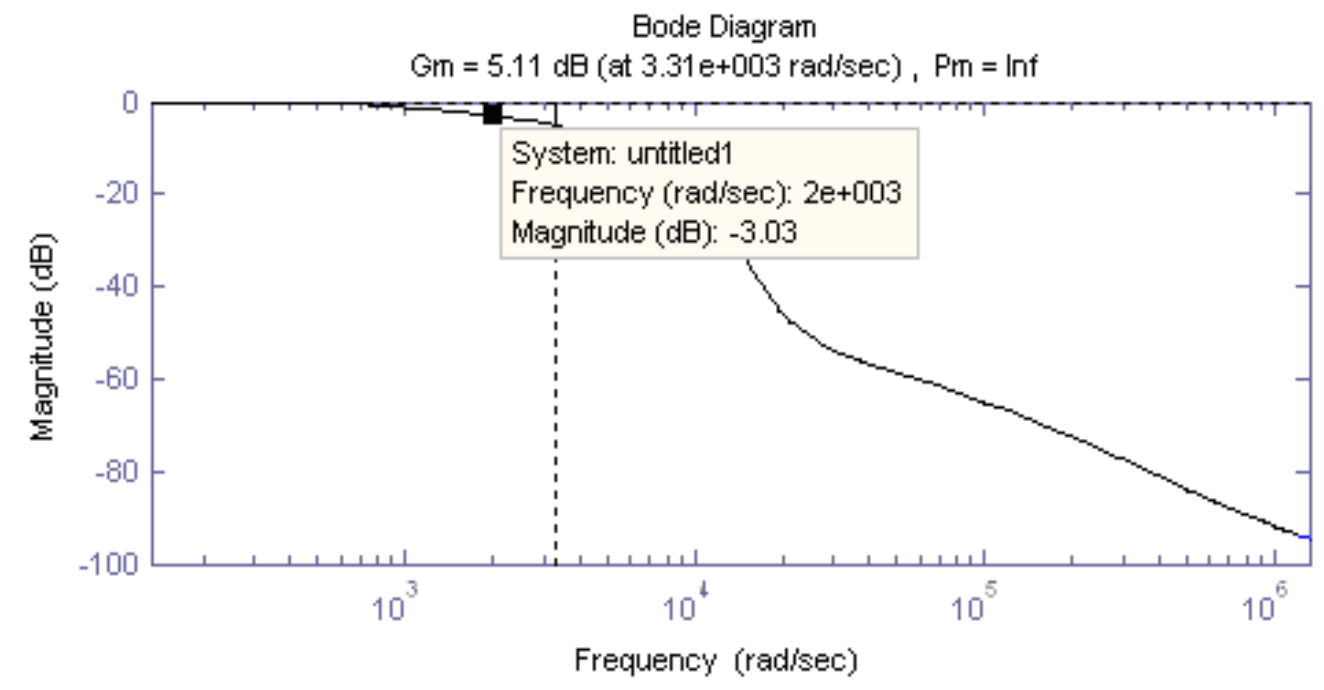

Figure 6. Closed-loop Magnitude-frequency Characteristic of the After-compensation Fine Tracking Subsystem

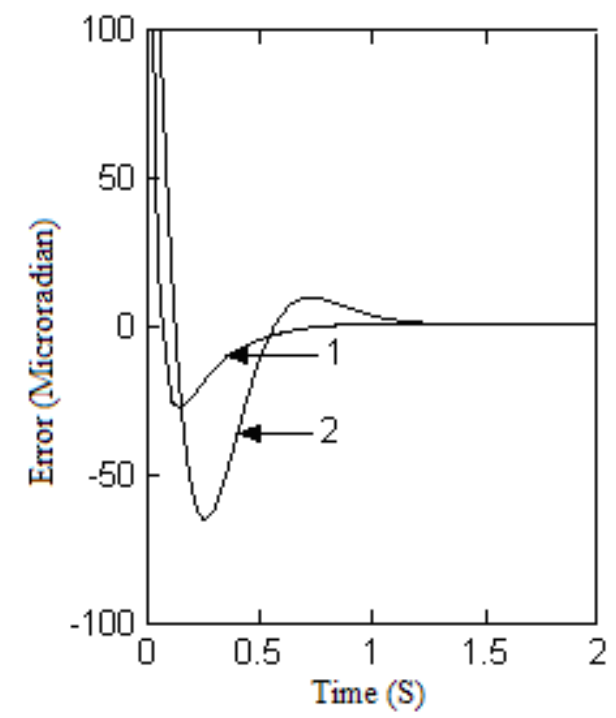

Figure 7. Equivalent Sine Simulation Result of APT System

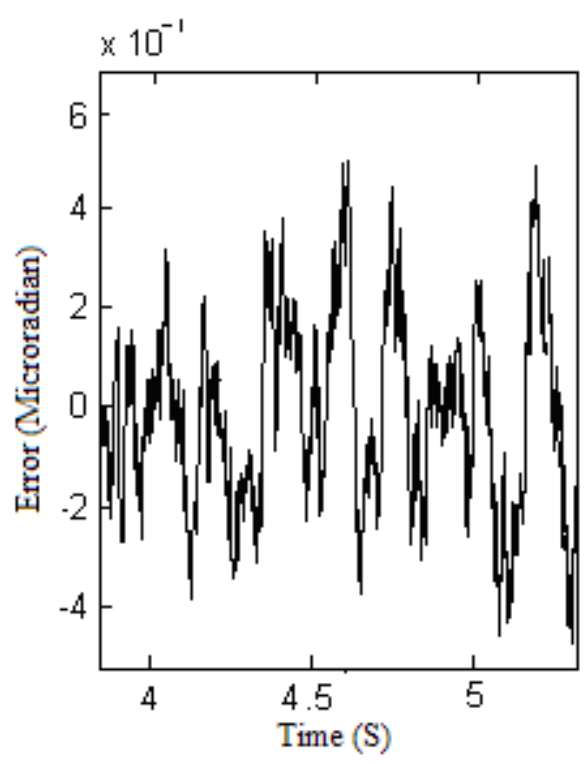

Figure 8. Part Enlargement of Error 